\title{
Is the rat prefrontal cortex crucial for cognitive control during spatial cognition?
}

\author{
Eun Hye Park ${ }^{\mathrm{a}, *}$, Kally C. O’Reilly ${ }^{\mathrm{a}, *, \#, \mathrm{~d}}$, David Taborga ${ }^{\mathrm{a}}$, Kyndall Nicholas ${ }^{\mathrm{a}}$, Armaan S Ahmed $^{\mathrm{a}}$, Natalie \\ Ruiza , André A. Fenton ${ }^{\mathrm{a}, \mathrm{b}, \mathrm{c}, \#}$ \\ ${ }^{a}$ Center for Neural Science, New York University, New York, NY 10003

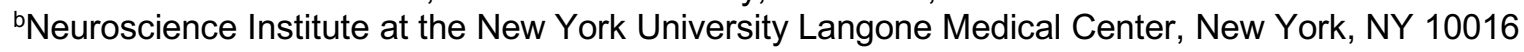 \\ 'Department of Physiology, SUNY Downstate Medical Center, Brooklyn, NY \\ ${ }^{\mathrm{d} C}$ Current address: Child and Adolescent Psychiatry, New York State Psychiatric Institute, 1051 Riverside \\ Drive, New York, NY 10032 \\ ${ }^{*}$ These authors contributed equally to this work \\ Author Contributions: KCO, EP, and AAF designed the research, KCO, EP, DT, KN, ASA, and NR \\ performed the research, EP and $\mathrm{KCO}$ analyzed the data and $\mathrm{AAF}, \mathrm{KCO}$, and $\mathrm{EP}$ wrote the paper. \\ \#Correspondence should be addressed to: \\ André A. Fenton \\ Center for Neural Science \\ New York University \\ 4 Washington Place \\ New York, NY 10003 \\ and \\ Kally C. O'Reilly \\ Child and Adolescent Psychiatry \\ New York State Psychiatric Institute \\ 1051 Riverside Dr. \\ New York, NY 10032 U.S.A.
}




\section{ABSTRACT}

Cognitive control tasks require that the subject use one class of information and ignore another competing class of information. In prior work, we used an active place avoidance task on a rotating arena that requires rodent subjects to avoid shock by using information about their location in the stationary room and ignore information about their location on the rotating floor. During the task, the discharge of hippocampus neurons alternates judiciously between representing stationary and rotating locations according to the proximity of shock, demonstrating cognitive control directly in the neural representations of hippocampal discharge. The central role of the medial prefrontal cortex (mPFC) in cognitive control is well established in the primate literature, and largely accepted in the rodent literature because mPFC damage causes deficits in tasks that may require cognitive control, as inferred, typically from the task design. Here we test whether rat mPFC lesion impairs the active place avoidance task that requires cognitive control in order to test the "central-computation" hypothesis in which the mPFC is hypothesized to be essential for the computations required for cognitive control. Although ibotenic acid lesion of the mPFC was effective and caused alterations in the coordination of metabolic activity, including the dorsal hippocampus to the dorsal subiculum, its output structure, nonetheless the lesion did not impair active place avoidance. These data support an alternative "local computation" hypothesis: the computations required for cognitive control can occur locally in brain networks independently of the MPFC as a central computational locus for cognitive control. 


\section{SIGNIFICANCE STATEMENT}

The medial prefrontal cortex (mPFC) is considered to be crucial for cognitive control of information, operating with winner-take-all dynamics that allows subjects to make judicious choices in the presence of alternatives. Alternatively, cognitive control may also result from computations in neural circuits such as hippocampus, with a neural architecture for winner-take-all computations. We investigated whether mPFC lesion impairs an active place avoidance task that is demonstrated to require cognitive control that is observed in hippocampus place cell spike train dynamics. We produced mPFC lesions with brain-wide consequences that reduced resting-state coordination of metabolic activity within hippocampus and related areas. Nonetheless mPFC lesion did not impair the active place avoidance task, demonstrating that cognitive control does not always depend on mPFC. 


\section{INTRODUCTION}

Cognitive control is the ability to judiciously use task-relevant information while ignoring salient concurrent information that is task irrelevant. This ability, also known as executive control, is crucial for completing tasks that require manipulation of complex knowledge and, because cognitive control is often impaired in neuropsychiatric disorders, it is a therapeutic target to improve clinical outcomes(Solomon et al., 2008; Lesh et al., 2011). The consensus view is that prefrontal cortex is crucial for cognitive control and other executive functions such that prefrontal cortical activity, especially maintained neural firing, is central to the underlying computations that operate through prefrontal interactions with diverse brain areas during cognitive control tasks(Miller and Cohen, 2001). Recent work has emphasized a central role for other brain areas such as sensory thalamus(Wimmer et al., 2015) and hippocampus(Kelemen and Fenton, 2010; Lee et al., 2012) in cognitive control-related interactions between modality-specific information processing neural circuits and control-specialized processing in the prefrontal cortex(Murray et al., 2017).

Although investigations of hippocampus function have traditionally emphasized spatial and memory-related information processing, recent work has explored the role of the hippocampus in the cognitive control of spatial and mnemonic information using an active place avoidance task. The task conditions rodents to avoid the location of a mild shock, and uses continuous rotation of the behavioral arena to dissociate the environment into two spaces, one defined by the rotating floor and the other defined by the stationary room. By selectively conditioning place avoidance responses to either shock at stationary room 
locations or shock at rotating arena locations, place learning tasks are configured to require cognitive control to judiciously use spatial information from the spatial frame that is conditioned and ignore spatial information from the other, task-irrelevant frame(Fenton et al., 1998). When both a stationary and a rotating shock zone are defined, inactivation of the dorsal hippocampus impairs task performance(Kelemen and Fenton, 2010). Importantly, the impairment is also observed when only the stationary shock zone is defined on the rotating arena, suggesting cognitive control is needed, not only to identify the locations of shock, but also to ignore the irrelevant arena locations of shock(Cimadevilla et al., 2000; Cimadevilla et al., 2001; Wesierska et al., 2005). Indeed, electrophysiological recordings of place cell activity in hippocampus subfields, as well as head-direction cell recordings from the medial entorhinal cortical input, confirm cognitive control of spatial representations because neural ensemble activity spontaneously alternates between representing room and arena locations depending on the rat or mouse's proximity to the frame-specific location of shock(Kelemen and Fenton, 2010; Talbot et al., 2018; van Dijk and Fenton, 2018; Park et al., 2019). This explicit observation of the dynamic functional grouping of neural activity into alternating neural representations of what is relevant during the place avoidance task is some of the most direct evidence of cognitive control, which is an internal process that is typically only inferred from the task demands and performance.

What, then, is the role of prefrontal cortex in active place avoidance tasks that require cognitive control? The consensus prefrontal hypothesis asserts that the prefrontal cortex is essential for the computations necessary for cognitive control. This "central- 
computation" hypothesis centralizes the necessary computations to the prefrontal cortex and predicts that lesion of the prefrontal cortex will be impairing. An alternative "localcomputation" hypothesis asserts that the computations needed for cognitive control can be performed locally in neural networks that are specialized for the particular information upon which the task depends and on which cognitive control operates. This hypothesis predicts that prefrontal lesion can be sparing of cognitive control. We tested these mutually exclusive hypotheses by lesion of the prefrontal cortex in rat subjects and tested whether they could learn the active place avoidance task.

\section{METHODS}

All methods complied with the Public Health and Service Policy on Humane Care and Use of Laboratory Animals and were approved by the Author's University Animal Welfare Committee, which follow National Institutes of Health guidelines.

\section{Subjects}

Twenty-four adult, male, Long-Evans rats were purchased from Charles River to arrive at New York University at approximately 40 days old. The rats were given at least one week to acclimate to the facility and were housed two per cage on a 12:12 light: dark cycle with free access to food and water. Twelve rats were assigned to the lesion group and 11 to the sham group. One animal was used to confirm the coordinates, by injecting fluorogold (Fluorochrome, Denver, $\mathrm{CO}, 2.5 \% \mathrm{w} / \mathrm{v}$ in distilled water) at the same rate and for the same duration as for the lesions. 
One rat in the lesion group was excluded because the lesion was inadequate and one had to be removed from the study due to a skin irritation, resulting in 10 rats in the lesion group. All of these rats underwent behavioral assessment (lesion $n=10$ ) but only a subset were stained for cytochrome oxidase (lesion $n=8$ ) because the tissue from one lesion and one sham rat was lost to thawing and tissue from one rat from each group was not processed. For the control group one rat was excluded from performing behavioral experiments because his lesioned cage mate had a skin irritation that prevented the lesion rat from behavioral testing; his brain was still processed for cytochrome oxidase activity. One rat was excluded from the behavioral experiments due to equipment issues during training, and one rat had tissue damage during the sham surgery that appeared as a lesion. Thus, the sham group had eight rats for behavioral assessment and eight for cytochrome oxidase assessment. Our final group numbers for the behavioral assessment were therefore sham group $n=8$, lesion group $n=10$ and our final group numbers for cytochrome oxidase activity were sham group $n=8$ and lesion group $n=8$.

\section{Surgery}

Between the ages of 48-56 days, the prefrontal cortex was lesioned under sodium pentobarbital (50 mg/kg, i.p.) anesthesia. Bilateral lesion occurred at two sites per hemisphere at the following stereotaxic coordinates: from Bregma, Site 1- A.P: $+2.5, M L$ : \pm 0.6 , D.V: -5.0 , relative to skull surface, Site 2-A.P : $+3.5, M L: \pm 0.6, D . V:-5.2$, relative to skull surface. Ibotenic acid was used to create an excitotoxic lesion (0.06 M in phosphate buffered saline) and was injected with a micro infusion pump at a flow rate 0.2 $\mu \mathrm{L} / \mathrm{min}$ through a stainless-steel cannula $(0.25 \mathrm{~mm}$ outer diameter). Sham group rats 
underwent the same surgery procedure, but phosphate buffered saline was injected. The rats were given one week to recover.

\section{Active place avoidance training}

After recovery from surgery, the rats were handled for 5 mins per day for 5 days. On the first day of behavior training, animals had a pretraining session that consisted of two trials during which the rats were allowed to explore the stationary arena to habituate the environment. The following two days, the rats underwent eight training trials per day on a rotating arena, learning to avoid entering a $60^{\circ}$ sector shock zone in which the rats received a mild foot shock $(500 \mathrm{~ms}, 60 \mathrm{~Hz}, 0.4 \mathrm{~mA})$. This shock zone was defined by the stationary cues in the room. On the fourth day of the behavioral training, the rats had a single trial with the shock on to test retention of the training. The rats were then trained on days 4 and 5 in a conflict training session in which the rats received eight trials per day with the shock zone located $180^{\circ}$ from the initial training position. All trials were $10 \mathrm{~min}$ with 10 min intertrial intervals.

\section{Tissue processing and histochemistry}

$\underline{\text { Verification of lesion coordinates }}$

Immediately following surgery to inject fluorogold, the rat was transcardially perfused with $0.9 \%$ saline and $10 \%$ formalin and the brain extracted and postfixed in $10 \%$ formalin for $24 \mathrm{~h}$ at $4^{\circ} \mathrm{C}$. The brain was then cryoprotected in $30 \%$ sucrose $(\mathrm{w} / \mathrm{v}$ in $1 \mathrm{x}$ phosphate buffered saline) and stored at $4^{\circ} \mathrm{C}$ until cut on a cryostat $(40 \mu \mathrm{m})$. The sections were mounted onto gelatin coated slides and scanned using an Olympus VS120 microscope 
(fluorescence, 10x). The slides were then placed in e-pure water (2 times, 1 min each) before being dehydrated (50,70, 80, 95, 100, 100\% ethanol, 1 min each) and defatted in 50:50 chloroform ethanol for $20 \mathrm{~min}$. The slides were then rehydrated $(100,100,90,80$, $70,50 \%$ ethanol, 1 min each) before being Nissl stained with cresyl violet (1 min), rinsed in e-pure, and dehydrated (50\% ethanol, $1 \mathrm{~min}, 70 \%$ ethanol until the white matter was white, 95\% ethanol with acetic acid until desired intensity, 100, 100\% ethanol, 2 min each). The tissues were then cleared in xylenes ( 3 times, 5 min each) before being coverslipped. The slides were then scanned again (light microscope, 10x). Images were manipulated using Adobe Photoshop CS6 to perform auto-contrast on each image and to remove background from the images. The fluorescent images were then all thresholded in a single operation to remove the background and the result was superimposed on the corresponding Nissl stained sections using Canvas 5.02.

\section{$\underline{\text { Cytochrome oxidase activity and Nissl staining }}$}

On the day following the completion of behavior training, the rats were anesthetized with isoflurane, immediately decapitated and the brains were extracted. The brains were rapidly frozen in isopentane on dry ice and stored at $-80^{\circ} \mathrm{C}$. Sets of brains consisting of two to four animals per group were cut simultaneously on a cryostat (40 $\mu \mathrm{m})$, and sorted into the three series, one of which was Nissl stained and one used for cytochrome oxidase histochemistry. The series used for Nissl staining was stored at room temperature and the other two series stored at $-80^{\circ} \mathrm{C}$ until processed for cytochrome oxidase histochemistry. To control for variability across batches of histochemical staining, 20, 40, 60 and $80 \mu \mathrm{m}$ sections of fresh rat brain tissue homogenate (prepared as in (Shumake et 
al., 2000) were included. Cytochrome oxidase staining was performed according to (O'Reilly et al., 2009). Stained slides were scanned with an Olympus VS 120 light microscope $(2 \mathrm{x})$ and the optical densities measured from captured images. Images were converted to 8-bit gray scale using imageJ $(\mathrm{NIH})$ and optical densities read from the standard slides and 14 brain regions (Figure 1) and included: dygranular and granular retrosplenial cortices (RSD and RSG, respectively), the nucleus reuniens (RE), the central nucleus of the amygdala (CEA), basomedial and basolateral amgydala (BMA, and BLA, respectively), the dorsal hippocampal CA1, CA2, CA3 and dentate gyrus areas (dCA1, dCA2, dCA3, dDG, respectively) the ventral hippocampal CA1, CA3 and dentate gyrus areas (vCA1,vCA3, vDG, respectively) and the dorsal subiculum (DS). The optical densities were measured using ImageJ $(\mathrm{NIH})$ and cytochrome oxidase activity was normalized as in (O'Reilly et al., 2016). Optical densities were measured while blind to the group identity. Three to six optical density readings were taken for each brain region, from both hemispheres and averaged for each individual subject.

To confirm the lesion site, one series of sections were stained with Cresyl violet. The slides were placed in the e-pure water (2 times, 1 min each) and dehydrated in a series of ethanol baths $(50,70,80,90,100,100 \%, 1$ min each) prior to clearing the fats in a $1: 1$ mixture of ethanol:chloroform for $20 \mathrm{~min}$. The slides were then rehydrated $(100,100,90$, $80,70,50 \%$ ethanol, 1 min each), rinsed in e-pure water, and placed in the Cresyl violet for $10 \mathrm{~min}$. The slides were again dehydrated and cleared in xylenes ( 3 times, 5 min each) before being coverslipped. Images were captured with an Olympus VS120 light 
microscope at 10x. Only rats with verified bilateral lesions were included in the data analysis.

\section{Statistical analysis}

Cytochrome oxidase activity: Group averages of the optical densities were calculated for each brain regions and expressed as mean \pm SEM relative cytochrome oxidase activity/ $\mu \mathrm{m}$ of tissue. Functional connectivity was examined by calculating Pearson correlations between each brain region. To determine if the interregional correlations were significantly different between groups, we transformed the $r$ value of the correlation to fisher's z-scores. Significant correlations $(p<0.05)$ were used to generate graph theoretical networks using the Brain Connectivity Toolbox in MATLAB (https://sites.google.com/site/bctnet/)

Behavior: Place avoidance task performance level was compared using multivariate analysis of variance. Statistical significance was set at 0.05 for all comparisons.

\section{RESULTS}

\section{Histological evaluation of mPFC lesion}

The mPFC lesions are summarized in Figure 1B. The ibotenic acid-induced lesions were restricted to mPFC and included the cingulate cortex, prelimbic and infralimbic areas. All animals included in further analyses had bilateral mPFC lesions (Figure 1B).

mPFC lesion can alter baseline functional relationships amongst related brain areas 
To evaluate whether the consequences of the lesion were restricted to mPFC, we measured the activity of cytochrome oxidase $(\mathrm{CO})$ in the brains of a subset of rats (lesion $n=8$, sham $n=8$ ). CO is a sensitive metabolic marker for neuronal function (Wong-Riley, 1989; O'Reilly et al., 2019), so to evaluate the consequence of mPFC lesion on brain function, CO activity was estimated in 14 functionally-related brain regions that included the dorsal and ventral hippocampus, amygdala, retrosplenial cortex and nucleus reunions (Figure 2D). We detected a group difference in the central amygdala with greater $\mathrm{CO}$ activity in the lesion rats (Table 1). We then examined the across-subject metabolic coupling between $\mathrm{CO}$ activity in pairs of these areas to evaluate whether mPFC lesion might alter functional relationships in the rest of the brain. The pattern of interregional correlations appears to differ between the lesion and sham groups, with some interregional correlations appearing weaker in the lesion group (Figure 2A). Univariate correlations amongst hippocampus, reunion, amygdala, and dorsal subiculum decreased after mPFC lesion, but these decreases did not survive the $p<0.0005$ Bonferroni correction for the 91 comparisons (Figure 2A; See legend or table 1 for abbreviations: BLA-RE: $z=1.91, p=0.05$, dCA1-CEA: $z=-3.12, p=0.001, d D G-d C A 3: z=2.79, p=$ 0.005, vCA1-RE: $z=1.94, p=0.05$, DS-RE: $z=1.92, p=0.05$, DS-dDG: $z=1.9, p=$ 0.05).

To further investigate whether mPFC lesion alters baseline covariations in metabolic function amongst the sample of brain areas, we applied network graph theoretical analyses with the 14 brain regions representing the nodes; the significant univariate interregional correlations $(p<0.05)$ represent the edges (Figure $2 B)$. After FDR correction 
(0.01) 15 of the 91 correlations were judged significant in the sham sample but only 7 in the lesion sample (test of proportions: $z=2.26, p=0.03$ ). The correlations within the ventral hippocampus were preserved but correlations to and amongst the dorsal hippocampus subfields were lost after mPFC lesion. These $\mathrm{CO}$ data indicate that the mPFC lesion caused functional changes beyond mPFC and specifically in hippocampus.

\section{mPFC lesions do not impair cognitive control in the active place avoidance task}

To assess the effect of mPFC lesion on behavior, we first examined the effect on spontaneous locomotion during the pretraining sessions. There is no effect of lesion on the total distance walked across the two sessions (Figure 3D; 2-way RM ANOVA F A $_{16}=$ $\left.0.04, p=0.85, \eta_{p}^{2}=0.002\right)$. There is an effect of trial $\left(F_{1,16}=15.31, p=0.001, \eta_{p}^{2}=0.49\right)$ but there is no effect of the interaction $\left(F_{1,16}=0.14, p=0.71, \eta_{p}{ }^{2}=0.01\right)$.

We next examined active place avoidance learning over the initial training period (Figure 3B, Days1-3). Both groups learned to reduce their entrances into the shock zone with training. There is no effect of group $\left(F_{1,16}=0.002, p=0.9, \eta_{p}^{2}=0.00\right)$, but effects of day $\left(F_{1,16}=26.34, p=10^{-4}, \eta_{p}^{2}=0.62\right)$ and trials $\left(F_{3.22,51.50}=36.76, p=10^{-13}, \eta_{p}^{2}=0.70\right)$ are significant. There are no effects of the group interactions (group $x$ day: $F_{1,16}=0.003, p=$ 0.96, $\eta_{p}^{2}=0.00 ;$ group $x$ trial: $F_{3.22,51.50}=0.74, p=0.54, \eta_{p}^{2}=0.00 ;$ group $x$ day $x$ trial: $\left.F_{2.26,36.08}=0.35, p=0.74, \eta_{p}^{2}=0.02\right)$. The interaction of day $x$ trial is significant $\left(F_{2.26}\right.$, $\left.36.08=13.75, p=10^{-5}, \eta_{p}^{2}=0.02\right)$. 
We also examined the latency to first enter the shock zone on each trial as this estimates between-trial learning without the confound of within-trial learning (Figure $3 \mathrm{C}$ ). Both groups increase their latencies to first enter the shock zone. There is no effect of group $\left(F_{1,16}=0.1, p=0.76, \eta^{2}=0.01\right)$ but clear effects of day $\left(F_{1,16}=69.61, p=10^{-7}, \eta^{2}=0.81\right)$ and trial $\left(F_{5.33,85.33}=12.15, p=10^{-9}, \eta_{p}^{2}=0.43\right)$. There are also no significant group interactions (group $x$ day: $F_{1,16}=0.94 ; p=0.35, \eta_{p}^{2}=0.06$; group $x$ trial: $F_{5.33,85.33}=1.83$, $p=0.09, \eta_{p}^{2}=0.10 ;$ group $x$ day $x$ trial: $\left.F_{4.78,76.43}=0.26, p=0.93, \eta_{p}^{2}=0.02\right)$. The day $x$ trial interaction is significant $\left(F_{4.78,76.43}=1.93, p=0.10, \eta_{p}{ }^{2}=0.11\right)$.

Twenty-four-hour memory retention was tested on day 4. The two groups are indistinguishable in their times to first enter the shock zone $\left(t_{16}=1.01, p=0.3, d=0.49\right)$ and the number of entrances $\left(t_{16}=0.95, p=0.4, d=0.002\right)$. There is no evidence that mPFC lesion causes a deficit in the active place avoidance task.

We then trained the rats to avoid the shock zone relocated $180^{\circ}$ to assess the impact of mPFC lesion on cognitive flexibility in this cognitive control challenge. Both groups learn the conflict task, measured by the number of entrances into the new shock zone (Figure 3B, Days 4-5). There is no effect of group $\left(F_{1,16}=0.21, p=0.65, \eta_{p}^{2}=0.01\right)$, but the effects of day $\left(F_{1,16}=8.99, p=0.01, \eta_{p}^{2}=0.36\right)$, and trials $\left(F_{1.89,30.12}=18.55, p=10^{-9}, \eta_{p}{ }^{2}=0.54\right)$ are significant. The group interactions are not significant (group $x$ day: $F_{1,16}=0.55, p=$ 0.47, $\eta_{p}{ }^{2}=0.36$; group $x$ trial: $F_{1.88,30.12}=1.38, p=0.27, \eta_{p}{ }^{2}=0.08 ;$ day $x$ trial: $F_{2.30,36.87}$ $=2.31, p=0.11 \eta_{p}{ }^{2}=0.13 ;$ group $x$ day $x$ trial: $\left.F_{2.30,36.87}=1.16, p=0.33, \eta_{p}{ }^{2}=0.07\right)$. The latency to first enter the new shock zone also increases for both groups (Figure 3C, days 
4-5). The effect of group is not significant $\left(F_{1,16}=0.21, p=0.65, \eta_{p}^{2}=0.01\right)$ but the effects of day $\left(F_{1,16}=20.46, p=10^{-3}, \eta_{p}^{2}=0.01\right)$ and trials $\left(F_{4.84,154.93}=10.94, p=10^{-8}, \eta_{p}{ }^{2}=\right.$ 0.002 ) are significant. There are also no significant interactions (group $x$ day: $F_{1,16}=0.03$, $p=0.86, \eta_{p}^{2}=0.002 ;$ group $x$ trial: $F_{4.02,64.38}=0.38, p=0.54, \eta_{p}{ }^{2}=0.05 ;$ day $x$ trial: $F_{4.05}$ $64.81=1.29, p=0.28, \eta_{p}^{2}=0.08 ;$ group $x$ day $x$ trial: $\left.F_{4.05,64.81}=0.5, p=0.76, \eta_{p}^{2}=0.03\right)$.

\section{DISCUSSION}

We find no evidence that permanent lesion of mPFC impairs active place avoidance learning or memory to avoid an initial or relocated location of shock, despite CO evidence that the lesion may reduce the functional coupling between related brain areas. We confirmed the extent of the lesion and, using $\mathrm{CO}$ imaging, independently confirmed the lesion caused changes beyond the directly damaged area. In particular, a metabolic increase was observed in the amygdala and changes in the covariation of metabolic activity was detected in the interregional correlations, especially reduced correlations within ventral and dorsal hippocampus and between them and other structures; presumably these changes are compensatory in response to loss of innervation caused by the lesion. Accordingly, these findings reject the central-computation hypothesis that the mPFC is essential for the neural computations that are necessary for cognitive control tasks. Parenthetically, we stress that the present findings do not rule out a role for mPFC and hippocampal interactions in other cognitive processes(Adhikari et al., 2010; Sigurdsson et al., 2010; Spellman et al., 2015; Negrón-Oyarzo et al., 2018), especially those that rely on ventral hippocamps and perhaps the direct mPFC-ventral hippocampal connections(Hoover and Vertes, 2007), as such questions are beyond the scope of the 
experimental design. Because numerous manipulations of dorsal hippocampus, including bilateral inactivation, and unilateral inactivation, impair learning, consolidation and retrieval of the conditioned avoidance(Cimadevilla et al., 2000; Cimadevilla et al., 2001; Jezek et al., 2002; Kubik and Fenton, 2005; Wesierska et al., 2005), the present data instead favor the local-computation hypothesis. Accordingly, the dorsal hippocampus has been demonstrated to do goal-directed representational switching that is a sine qua non for cognitive control of spatial information(Kelemen and Fenton, 2010, 2016; Dvorak et al., 2018; Talbot et al., 2018; van Dijk and Fenton, 2018). More targeted hippocampal manipulations like X-irradiation and genetic ablation of adult neurogenesis(Burghardt et al., 2012), and optogenetic inactivation of granule cells(Kheirbek et al., 2013; Talbot et al., 2018) spare learning to avoid the initial location of shock in the active place avoidance task, but impair conflict learning to avoid the relocated location of the shock, unlike lesion of mPFC, as observed in the present study. Global manipulations such as genetic deletion of calbindin to promote aging(Moreno et al., 2011), as well as less specific manipulations, such as transient global cerebral ischemia to cause CA1 lesion(Popp et al., 2011), also impair active place avoidance learning, whereas mPFC lesion does not. It is certainly possible that acute manipulations of mPFC, such as optogenetic, chemogenetic or pharmacological inactivation, would have led to different results, suggesting that postlesion reorganization of neural circuits is sufficient to compensate for the loss of the mPFC functions that support cognitive control as required by the active place avoidance task. Nonetheless, this would not change the conclusion to reject the central-computation hypothesis. It is also possible that the role of the mPFC is best evaluated in tasks that rely on egocentric spatial information(Kesner et al., 1989), rather than the allocentric 
spatial information upon which the present active place avoidance task variant relies, but this very fact would also require rejecting, or at least severely limiting the centralcomputation hypothesis.

Taken together these data are difficult to explain on the central-computation hypothesis that the mPFC is essential for cognitive control, but the findings are consistent with the local-computation hypothesis that diverse (but probably not all) brain circuits can perform the computations needed for cognitive control of the information and/or behavior that the circuit is specialized to process. 


\section{REFERENCES}

Adhikari A, Topiwala MA, Gordon JA (2010) Synchronized activity between the ventral hippocampus and the medial prefrontal cortex during anxiety. Neuron 65:257-269. Burghardt NS, Park EH, Hen R, Fenton AA (2012) Adult-born hippocampal neurons promote cognitive flexibility in mice. Hippocampus 22:1795-1808.

Cimadevilla JM, Fenton AA, Bures J (2000) Functional inactivation of dorsal hippocampus impairs active place avoidance in rats. Neurosci Lett 285:53-56.

Cimadevilla JM, Wesierska M, Fenton AA, Bures J (2001) Inactivating one hippocampus impairs avoidance of a stable room-defined place during dissociation of arena cues from room cues by rotation of the arena. Proc Natl Acad Sci U S A 98:3531-3536.

Dvorak D, Radwan B, Sparks FT, Talbot ZN, Fenton AA (2018) Control of recollection by slow gamma dominating mid-frequency gamma in hippocampus CA1. PLoS Biol 16:e2003354.

Fenton AA, Wesierska M, Kaminsky Y, Bures J (1998) Both here and there: simultaneous expression of autonomous spatial memories in rats. Proc Natl Acad Sci U S A 95:11493-11498.

Hoover WB, Vertes RP (2007) Anatomical analysis of afferent projections to the medial prefrontal cortex in the rat. Brain Struct Funct 212:149-179.

Jezek K, Wesierska M, Fenton AA (2002) Hippocampus-dependent retrieval and hippocampus-independent extinction of place avoidance navigation, and stressinduced out-of-context activation of a memory revealed by reversible lesion experiments in rats. Physiol Res 51 Suppl 1:S35-47. 
Kelemen E, Fenton AA (2010) Dynamic grouping of hippocampal neural activity during cognitive control of two spatial frames. PLoS Biol 8:e1000403.

Kelemen E, Fenton AA (2016) Coordinating different representations in the hippocampus. Neurobiol Learn Mem 129:50-59.

Kesner RP, Farnsworth G, DiMattia BV (1989) Double dissociation of egocentric and allocentric space following medial prefrontal and parietal cortex lesions in the rat. Behav Neurosci 103:956-961.

Kheirbek MA, Drew LJ, Burghardt NS, Costantini DO, Tannenholz L, Ahmari SE, Zeng H, Fenton AA, Hen R (2013) Differential Control of Learning and Anxiety along the Dorsoventral Axis of the Dentate Gyrus. Neuron 77:955-968.

Kubik S, Fenton AA (2005) Behavioral evidence that segregation and representation are dissociable hippocampal functions. J Neurosci 25:9205-9212.

Lee H, Dvorak D, Kao HY, Duffy AM, Scharfman HE, Fenton AA (2012) Early cognitive experience prevents adult deficits in a neurodevelopmental schizophrenia model. Neuron 75:714-724.

Lesh TA, Niendam TA, Minzenberg MJ, Carter CS (2011) Cognitive control deficits in schizophrenia: mechanisms and meaning. Neuropsychopharmacology 36:316338.

Miller EK, Cohen JD (2001) An integrative theory of prefrontal cortex function. Annu Rev Neurosci 24:167-202.

Moreno H, Burghardt NS, Vela-Duarte D, Masciotti J, Hua F, Fenton AA, Schwaller B, Small SA (2011) The absence of the calcium-buffering protein calbindin is 
associated with faster age-related decline in hippocampal metabolism. Hippocampus.

Murray JD, Bernacchia A, Roy NA, Constantinidis C, Romo R, Wang XJ (2017) Stable population coding for working memory coexists with heterogeneous neural dynamics in prefrontal cortex. Proc Natl Acad Sci U S A 114:394-399.

Negrón-Oyarzo I, Espinosa N, Aguilar-Rivera M, Fuenzalida M, Aboitiz F, Fuentealba P (2018) Coordinated prefrontal-hippocampal activity and navigation strategyrelated prefrontal firing during spatial memory formation. Proceedings of the National Academy of Sciences of the United States of America 115:7123-7128.

O'Reilly KC, Perica MI, Fenton AA (2016) Memory deficits with intact cognitive control in the methylazoxymethanol acetate (MAM) exposure model of neurodevelopmental insult. Neurobiol Learn Mem 134 Pt B:294-303.

O'Reilly KC, Perica MI, Fenton AA (2019) Synaptic plasticity/dysplasticity, process memory and item memory in rodent models of mental dysfunction. Schizophr Res. O'Reilly KC, Shumake J, Bailey SJ, Gonzalez-Lima F, Lane MA (2009) Chronic 13-cisretinoic acid administration disrupts network interactions between the raphe nuclei and the hippocampal system in young adult mice. Eur J Pharmacol 605:68-77.

Park EH, Keeley S, Savin C, Ranck JB, Jr., Fenton AA (2019) How the Internally Organized Direction Sense Is Used to Navigate. Neuron.

Popp SS, Lei B, Kelemen E, Fenton AA, Cottrell JE, Kass IS (2011) Intravenous antiarrhythmic doses of lidocaine increase the survival rate of CA1 neurons and improve cognitive outcome after transient global cerebral ischemia in rats. Neuroscience 192:537-549. 
Shumake J, Poremba A, Edwards E, Gonzalez-Lima F (2000) Congenital helpless rats as a genetic model for cortex metabolism in depression. Neuroreport 11:37933798.

Sigurdsson T, Stark KL, Karayiorgou M, Gogos JA, Gordon JA (2010) Impaired hippocampal-prefrontal synchrony in a genetic mouse model of schizophrenia. Nature 464:763-767.

Solomon M, Ozonoff SJ, Cummings N, Carter CS (2008) Cognitive control in autism spectrum disorders. Int J Dev Neurosci 26:239-247.

Spellman T, Rigotti M, Ahmari SE, Fusi S, Gogos JA, Gordon JA (2015) Hippocampalprefrontal input supports spatial encoding in working memory. Nature 522:309-314.

Talbot ZN, Sparks FT, Dvorak D, Curran BM, Alarcon JM, Fenton AA (2018) Normal CA1 Place Fields but Discoordinated Network Discharge in a Fmr1-Null Mouse Model of Fragile X Syndrome. Neuron 97:684-697.

van Dijk MT, Fenton AA (2018) On How the Dentate Gyrus Contributes to Memory Discrimination. Neuron 98:832-845.

Wesierska M, Dockery C, Fenton AA (2005) Beyond memory, navigation, and inhibition: behavioral evidence for hippocampus-dependent cognitive coordination in the rat. J Neurosci 25:2413-2419.

Wimmer RD, Schmitt LI, Davidson TJ, Nakajima M, Deisseroth K, Halassa MM (2015) Thalamic control of sensory selection in divided attention. Nature 526:705-709. Wong-Riley MT (1989) Cytochrome oxidase: an endogenous metabolic marker for neuronal activity. Trends Neurosci 12:94-101. 


\section{LEGENDS}

Figure 1. Assessing the role of the mPFC in cognitive control and flexibility. A) Rats with mPFC or sham lesions were tested for cognitive ability using the two-frame active place avoidance task. Training to an initial shock zone (Initial training) estimated cognitive control, the ability to ignore the irrelevant cues associated with the rotating arena while tending to the stationary room cues that indicate where the shock zone is. Retention was used to assess memory of the shock zone after two days of training, and cognitive flexibility was assessed by relocating the shock zone by $180^{\circ}$ (conflict training). The impact of mPFC lesion on brain networks was examined by histological estimation of cytochrome oxidase activity, a metabolic marker of neuronal activity. Functional connectivity between brain regions was estimated by calculating the interregional Pearson correlations. B) To determine the appropriate coordinates for injection of ibotenic acid, we first injected fluorogold and examined the extent of fluorescent labeling (left column). mPFC and sham lesions were evaluated in Nissl stained histological sections and mapped onto a standard brain from the Rat Stereotaxic Coordinates Atlas (center column). The right column shows a representative mPFC from a sham rat (top) and a rat after ibotenic acid lesions (bottom). PrL(prelimbic), IL(infralimbic), Cg (cingulate cortex).

Figure 2. mPFC lesions change resting-state metabolic functional connectivity between the dorsal and ventral hippocampus. A) The matrix of cytochrome oxidase activity correlations amongst brain regions indicates that mPFC lesions reduce Pearson correlations in the lesion group. B) Graph theoretical analyses were used to visualize differences between the sham (left) and lesioned (right) groups. Each line in a network 
represents a significant correlation ( $p<0.05)$ between the two brain regions ("nodes") it connects; only the red lines survived False Discovery Rate (FDR < 0.01) correction. The degree of connectivity for each node was determined by counting the number of significant correlations from each node to all the other nodes in the network. Sham: $\mathrm{n}=$ 8; Lesion: $\mathrm{n}=8$. Functional connectivity in the groups differ $p<0.05$.

C) The number of significantly connected nodes at each brain area, before FDR correction. D) Representative cytochrome oxidase staining and locations of the optical density readings. Cytochrome oxidase activity was measured in the dygranular and granular retrosplenial cortices (RSD and RSG, respectively), the nucleus reuniens (RE), the central nucleus of the amygdala (CEA), basomedial and basolateral amygdala (BMA, and BLA, respectively), the dorsal hippocampal CA1, CA2, CA3 and dentate gyrus areas (dCA1, dCA2, dCA3, dDG, respectively) the ventral hippocampal CA1, CA2 and CA3 areas (vCA1, vCA3, vDG, respectively) and the dorsal subiculum (DS) marked as circles.

Figure 3. No detectable impact of mPFC lesion on cognitive control and flexibility measured in the active place avoidance task. A) Rats were trained in the two-frame active place avoidance task over the course of five days. Pretraining: On day 1, the rats were introduced to the stationary arena through two habituation trials. Initial Training: On days 2 and 3 the rats were trained to avoid a $60^{\circ}$ sector of the arena in which they would receive a mild foot shock. Each day the rats received eight training trials. Retention: Rats were tested in a single trial to the initial shock zone on day 4 . Afterwards the rats were given Conflict Training for two days (days 4 and 5), in which rats were given eight trials per day 
to learn to avoid a new shock zone located $180^{\circ}$ from the initial shock location. All trials were 10 min with a 10 min inter-trial interval. B) mPFC lesion rats are not different from sham rats in their ability to learn to avoid the shock zone, as indicated by the reduction of entrances into the shock zone over the Initial Training (group: $F_{1,16}=0.002, p=0.9$; day: $F_{1,16}=26.34, p=10^{-4}$; trials: $\left.F_{3.22,51.50}=36.76, p=10^{-13}\right)$. There is a significant interaction of day $x$ trials (group $x$ day: $F_{1,16}=0.003, p=0.96$; group $x$ trials: $F_{3.22,51.50}=0.74, p=$ 0.54; day $x$ trials: $F_{2.26,36.08}=13.75, p=10^{-5}$, group $x$ day $x$ trials: $F_{2.26,36.08}=0.35, p=$ 0.74). The lesion group is not different from the sham group in their retention of the shock zone $24 \mathrm{~h}$ after training $\left(\mathrm{t}_{16}=1.01, \mathrm{p}=0.3\right)$. In the conflict training, with the shock zone relocated $180^{\circ}$, there are no differences between lesion and sham rats $\left(F_{1,16}=0.21, p=\right.$ 0.65 ) but there is a significant effect of training day and trials (day: $F_{1,16}=8.99, p=0.01$; trials: $\left.F_{1.89,30.12}=18.55, p=10^{-9}\right)$. There are no significant interactions (group $x$ day: $F_{1,16}$ $=0.55, p=0.47$; group $x$ trial: $F_{1.88,30.12}=1.38, p=0.27 ;$ day $x$ trial: $F_{2.30,36.87}=2.31, p=$ 0.11 ; group $x$ day $x$ trial: $\left.F_{2.30,36.87}=1.16, p=0.33\right)$. C) There is no difference in the ability of sham and lesion rats to remember the shock zone, as seen by their latency to enter the shock zone for the first time on each trial. Both groups increase the time to enter the shock zone over training (group: $F_{1,16}=0.1, p=0.76$, day: $F_{1,16}=69.61, p=10^{-7}$, trial: $\left.\mathrm{F}_{5.33,85.33}=12.15, \mathrm{p}=10^{-9}\right)$ without significant interactions between the factors $\left(\mathrm{F}_{1,16}=\right.$ 0.94; $p=0.35 ;$ group $x$ trial: $F_{5.33,85.33}=1.83, p=0.09 ;$ group $x$ day $x$ trial: $F_{4.78,76.43}=$ 0.26, $p=0.93$; day $x$ trial: $\left.F_{4.78,76.43}=1.93, p=0.10\right)$. D) Lesion rats have normal locomotor activity, measured by the distance walked during pretraining (group: $F_{1,16}=$ 0.04, $p=0.85$; trials: $\left.F_{1,16}=15.31, p=0.001\right)$. For $B-D$, Repeated measure analyses were performed using the Greenhouse-Geisser correction. 
A

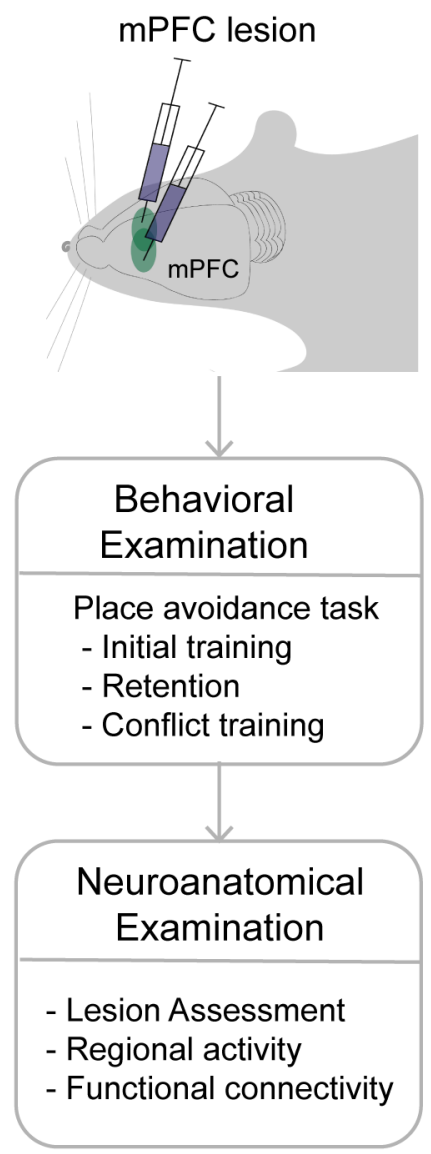

B

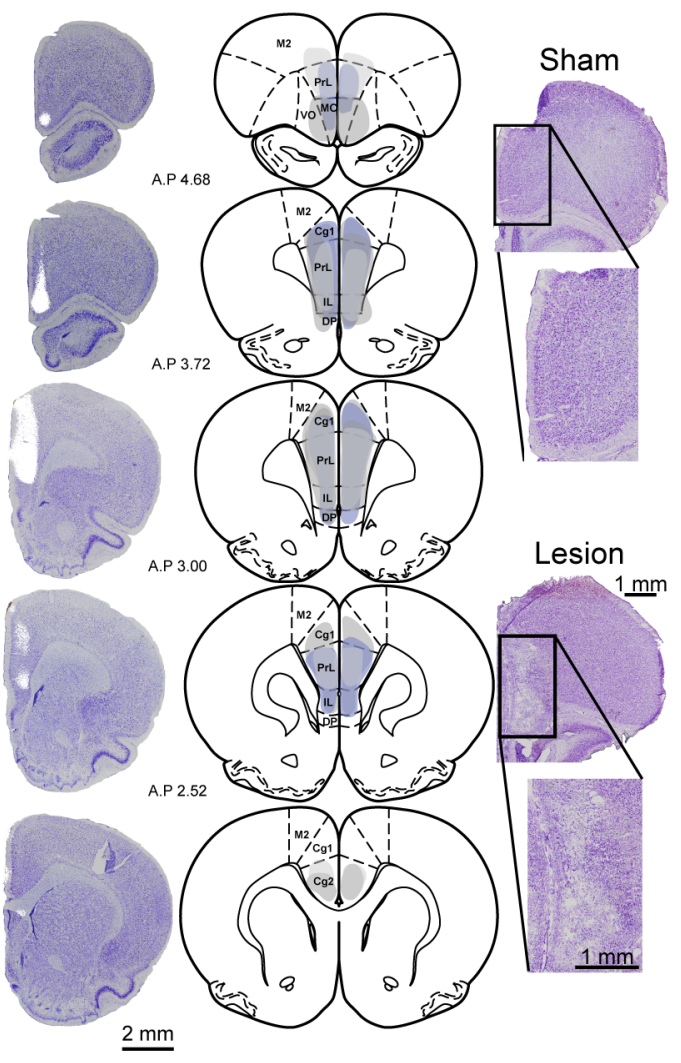

Figure 1 
bioRxiv preprint doi: https://doi.org/10.1101/2019.12.20.884262; this version posted December 22, 2019. The copyright holder for this preprint (which was not certified by peer review) is the author/funder, who has granted bioRxiv a license to display the preprint in perpetuity. It is made available under aCC-BY-ND 4.0 International license.

A

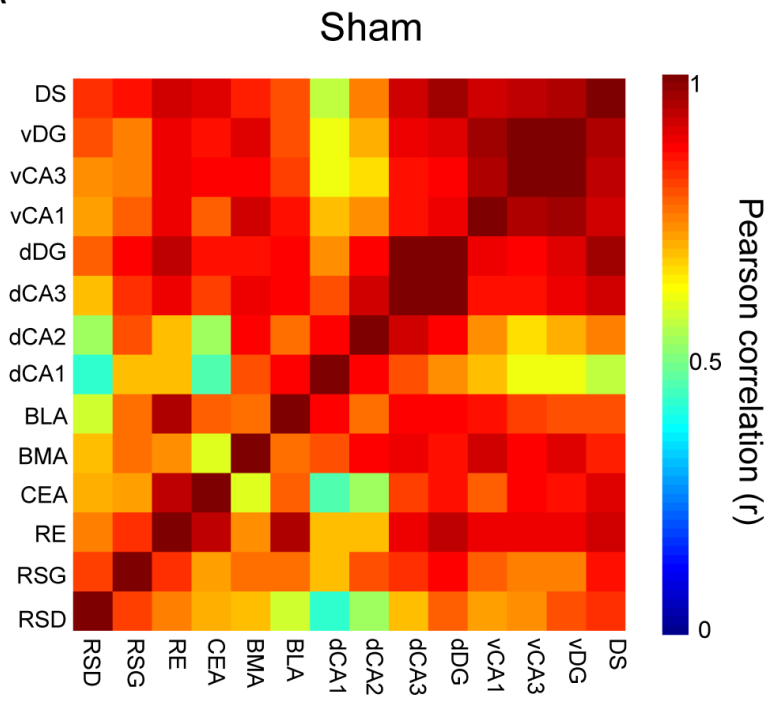

B

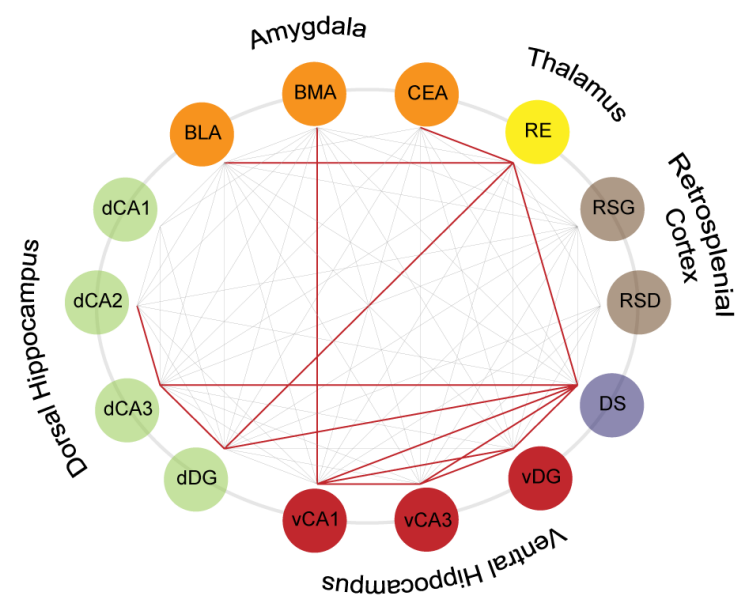

Lesion

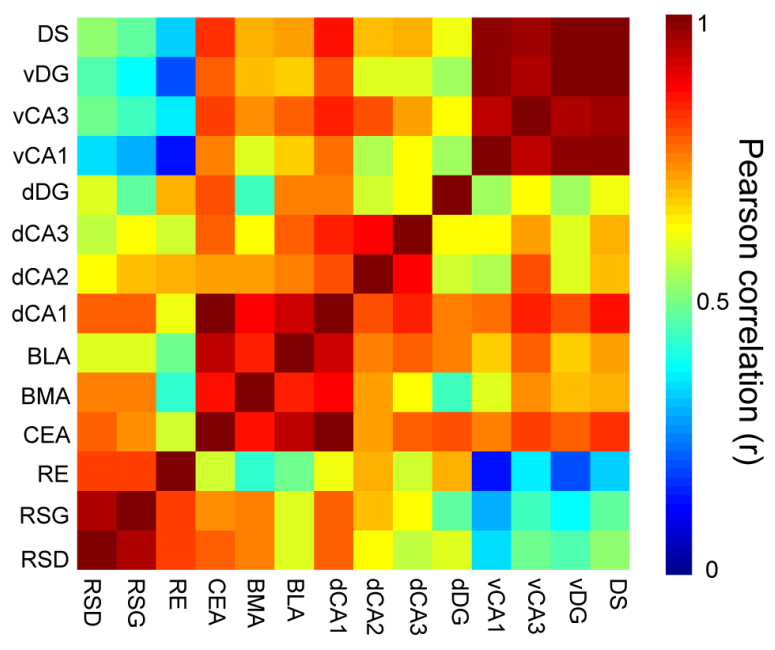

\section{Lesion}

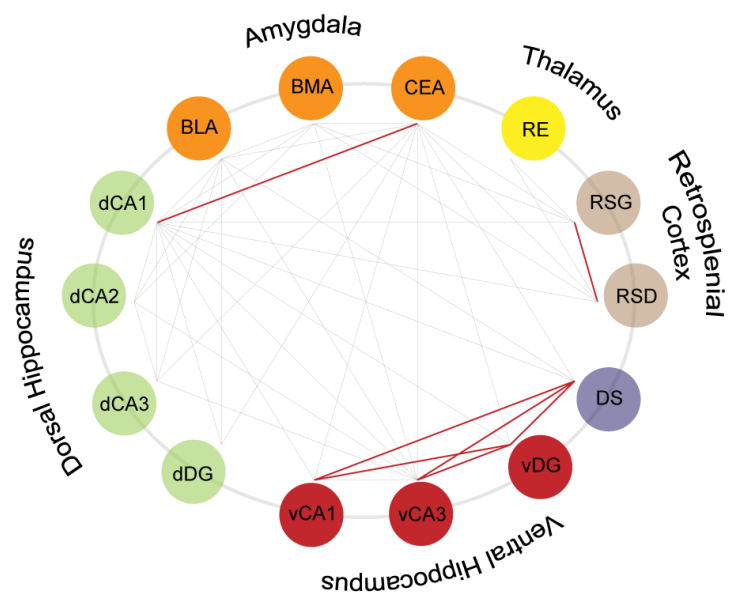

C

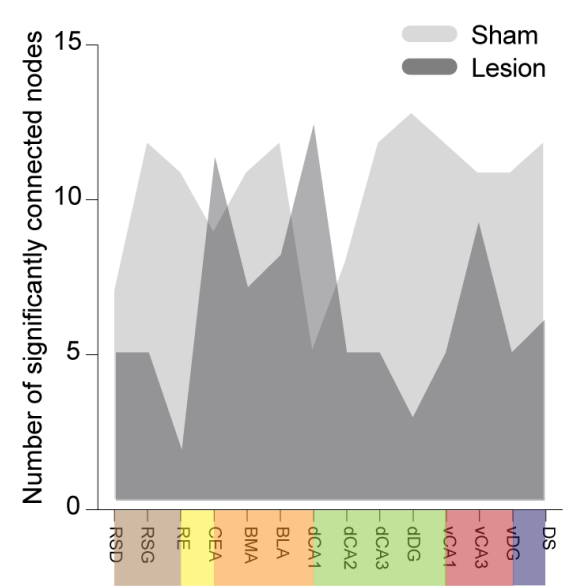

D

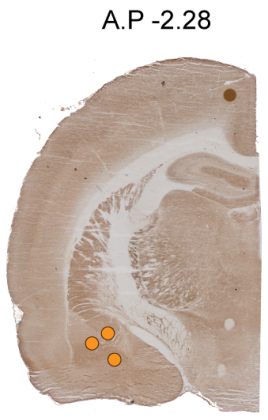

Retrosplenial Cortex

Amygdala

Nucleus Reuniens

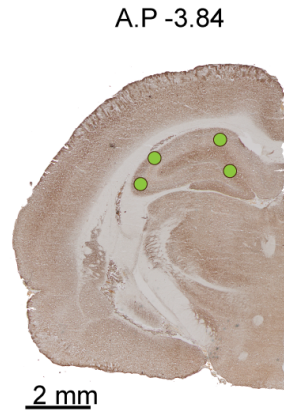

Dorsal

Hippocampus

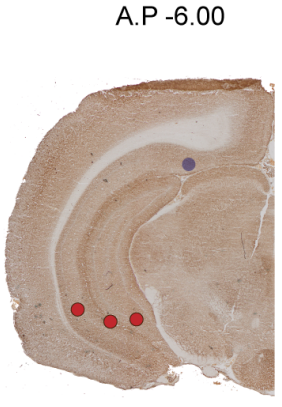

Ventral

Hippocampus Dorsal Subiculum

Figure 2 
A
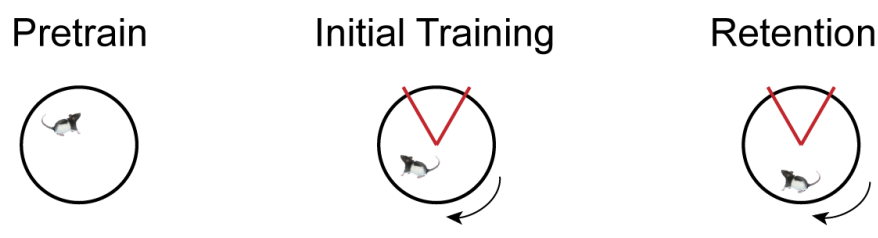

Conflict Training
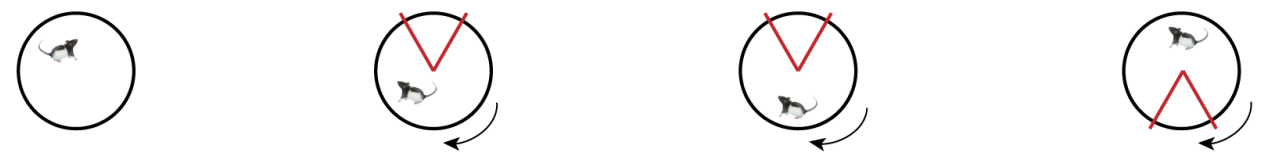

B

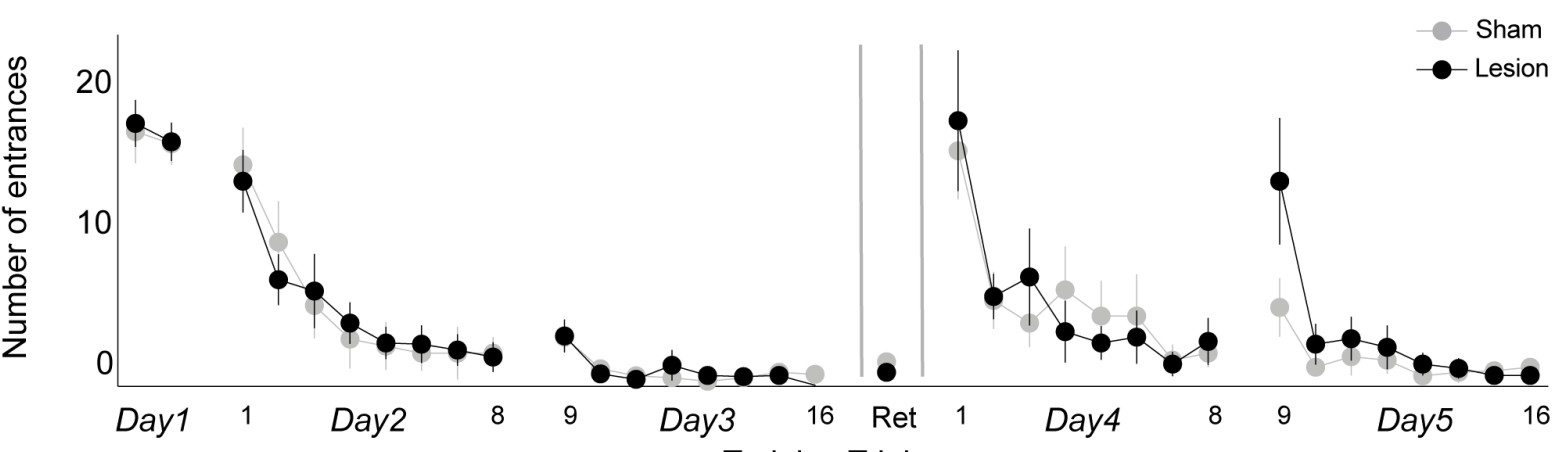

C

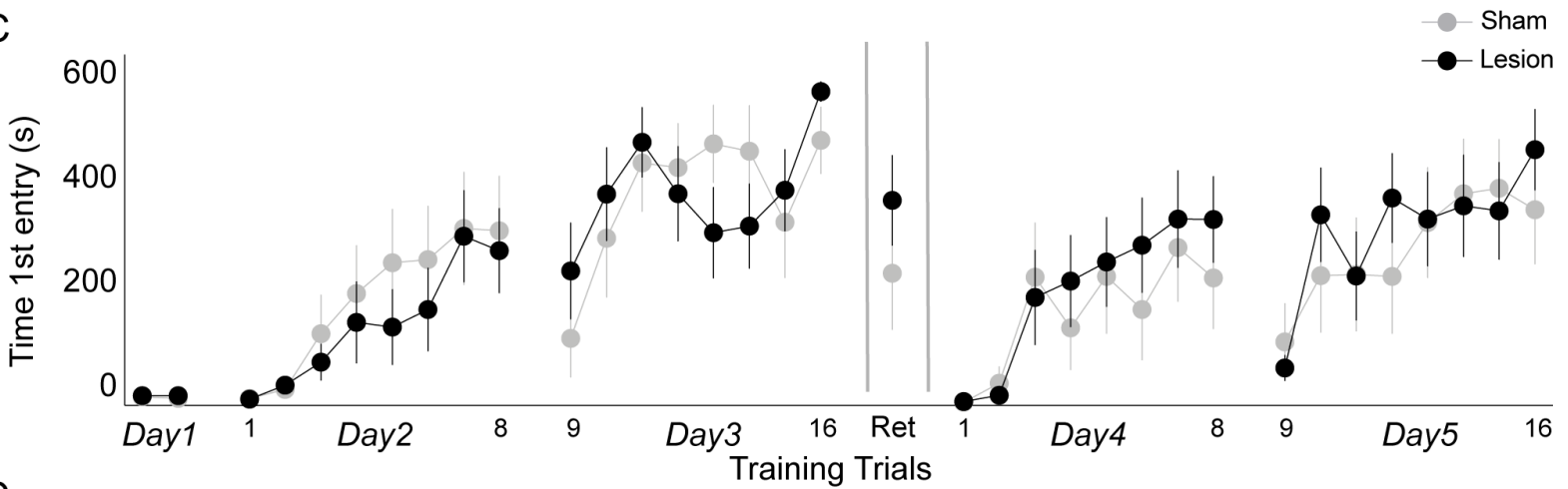

$\mathrm{D}$

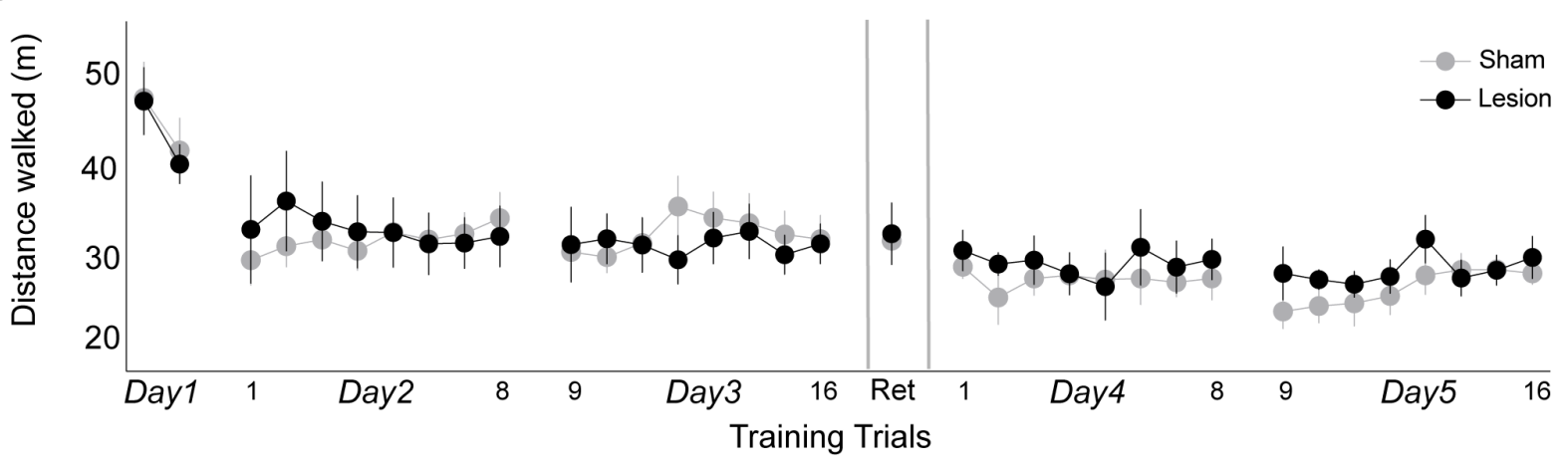

Figure 3 
Table 1

Average Cytochrome oxidase activity by brain region between Sham and Lesion groups

Relative Co activity / $\mu \mathrm{m}$ tissue $\left(\times 10^{-1}\right)$

\begin{tabular}{ccccc}
\hline Brain Region & $\begin{array}{c}\text { Sham } \\
\text { (Ave } \pm \text { SEM })\end{array}$ & $\begin{array}{c}\text { Lesion } \\
\text { (Ave } \pm \text { SEM })\end{array}$ & t ratio & p-value \\
\hline RSD & $1.84 \pm 0.09$ & $2.01 \pm 0.14$ & 1.04 & 0.32 \\
RSG & $1.91 \pm 0.09$ & $2.16 \pm 0.09$ & 1.86 & 0.08 \\
RE & $1.47 \pm 0.08$ & $1.66 \pm 0.09$ & 1.51 & 0.15 \\
CEA & $1.27 \pm 0.18$ & $1.94 \pm 0.19$ & 2.61 & $0.02^{*}$ \\
BMA & $1.31 \pm 0.11$ & $1.54 \pm 0.20$ & 0.99 & 0.34 \\
BLA & $1.33 \pm 0.17$ & $1.72 \pm 0.20$ & 1.49 & 0.16 \\
dCA1 & $1.51 \pm 0.08$ & $1.60 \pm 0.04$ & 0.97 & 0.35 \\
dCA2 & $1.34 \pm 0.08$ & $1.43 \pm 0.06$ & 0.98 & 0.34 \\
dCA3 & $1.50 \pm 0.10$ & $1.63 \pm 0.10$ & 0.94 & 0.36 \\
dDG & $1.39 \pm 0.08$ & $1.50 \pm 0.10$ & 0.85 & 0.41 \\
vCA1 & $1.54 \pm 0.15$ & $1.74 \pm 0.14$ & 1.02 & 0.33 \\
vCA3 & $1.73 \pm 0.14$ & $1.92 \pm 0.15$ & 0.94 & 0.36 \\
vDG & $1.75 \pm 0.12$ & $1.92 \pm 0.13$ & 0.92 & 0.37 \\
DS & $1.36 \pm 0.14$ & $1.61 \pm 0.19$ & 1.03 & 0.32 \\
\hline
\end{tabular}

RSD: dygranular retrosplenial cortices, RSG: granular retrosplenial cortices, RE: the nucleus reuniens, CEA: the central nucleus of the amygdala, BMA: basomedial amygdala BLA: basolateral amygdala, dCA1: the dorsal CA1, dCA2: dorsal CA2, dCA3: dorsal CA3 dDG: dorsal dentate gyrus, vCA1: ventral CA1, vCA3: ventral CA3, vDG : ventral dentate gyrus, and DS: dorsal subiculum. (Sham; $n=8$, Lesion; $n=8$ ) 